\title{
Microscopic Polyarteritis
}

National Cancer Institute

\section{Source}

National Cancer Institute. Microscopic Polyarteritis. NCI Thesaurus. Code C70549.

A systemic necrotizing vasculitis that typically affects the small and medium-sized

muscular arteries. In some cases, however, microscopic vessels are also affected (e.g., in the kidneys), a condition that has been called microscopic polyarteritis or polyang iitis; this disorder is felt to be more closely associated with Wegener granulomatosis than to classic polyarteritis nodosa. 